\title{
Studies of Natural Electric and Magnetic Fields ${ }^{1}$
}

\author{
G. D. Garland and T. F. Webster
}

(February 26, 1960)

\begin{abstract}
Simultaneous measurements of short-period natural electric field variations across western Canada are reported. From these it is indicated that the effect of the varying depth to the Precambrian rocks is the dominant factor. Analysis of the simultaneous magnetic and electric measurements gives a resistivity for the Precambrian basement in excess of $30 \times 10^{5}$ ohm meters.
\end{abstract}

\section{Introduction}

In considering the possibilities of earth-current communication, a knowledge of natural electric fields in the earth would appear to have significance for two reasons. First, because the natural effects form a background of noise above which an artificial signal must be detected, and secondly, because measurements of natural fields offer a method of deducing the electrical properties, in place, of portions of the earth's crust.

It has been recognized for some time that natural electric currents in the earth, as detected by measurement of the tangential electric field at the surface, bear a relationship to magnetic disturbances. Recently, Hessler and Wescott [1] ${ }^{2}$ have shown the variation in magnitude of the electric field as a function of the magnetic $K$-index at College, Alaska. However, the pricise relationship between the surface electric and magnetic disturbance fields at some point on the earth depends on both the distribution of sources and on the variation of conductivity within the earth. It is only if these effects can be separated that useful information on conductivities can be obtained.

An important simplification is possible if the source of disturbance is extended laterally, so that the arrival at the earth's surface may be approximated by a plane wave. It is well known that the tangential electric and magnetic fields, in perpendicular azimuths, say $E_{x}$ and $H_{y}$, bear a simple relationship at the boundary of a uniform conductor. Then-

$$
E_{x} / H_{y}=(2 \sigma T)^{-\frac{1}{2}}
$$

where $\sigma$ is the conductivity and $T$ is the period of the disturbance. Cagniard [2] has suggested that this approximation is valid for the earth, and that measurements of the fields will lead to a useful effective or apparent conductivity. However, Wait [3] has pointed out that departures from plane-

1 Contribution from Department of Physies, University of Alberta, Edmonton, Alberta, Canada; paper presented at Conference on the Propagation of ELF Radio Waves, Boulder, Colo., Jan, 25, 1960. The experimental work was supported by the National Research Council of Canada.

2 Figures in brackets indicate the literature references at the end of this paper. wave conditions can be treated by a series expansion for $E_{x} / H_{y}$. Retaining the first two terms gives

$$
\begin{aligned}
E_{x} / H_{y}=\eta+\eta / 2 \gamma^{2} H_{y}\left[\partial^{2} H_{y} / \partial y^{2}-\partial^{2} H_{y} / \partial x^{2}\right. & \\
& \left.+2 \partial^{2} H_{x} / \partial_{x} \partial y\right]
\end{aligned}
$$

where $\gamma=\mathrm{i} \sigma \mu \omega-\epsilon \mu \omega^{2}$ is the intrinsic propagation constant of the earth, and $\eta=i \mu \omega / \gamma$ is its characteristic impedance. Additional terms in the series involve higher inverse powers of $\gamma$, where $1 / \gamma$ is characteristically some tens of kilometers. The simplified expression of eq (1) will be valid if the second derivatives involved in eq (2) are sufficiently small.

\section{Regional Telluric Measurements}

It is obviously important to know the extent to which the fields may vary with position on the earth as a result of changing source distance. In order to examine this, and also the effect of variations in earth conductivities, we have compared earth-current disturbances at a number of points across western Canada (fig. 1). The Dominion Observatory maintains an earth-current and magnetic recording station at Meanook, and observations on the electric

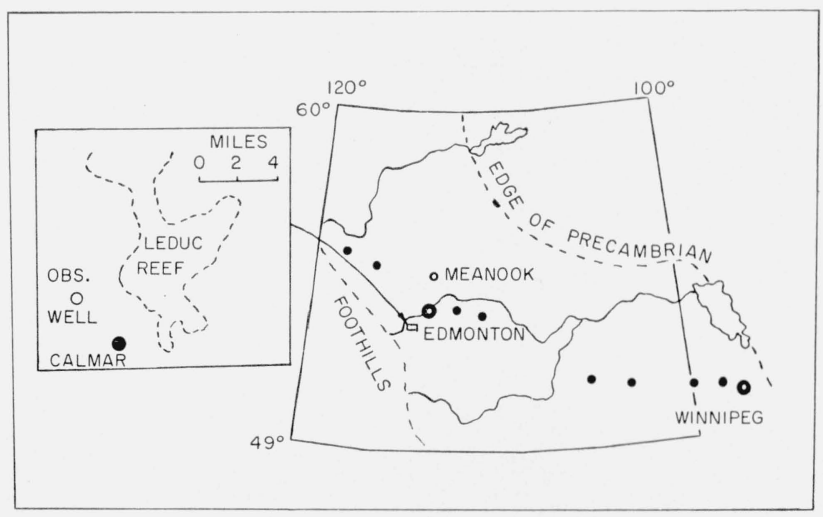

FIGURE 1. Location of telluric observation points, indicated by solid circles, and the Meanook magnetic observatory. Inset, location of well where vertical currents have been detected. 
field variations at the other points shown could be compared with simultaneous values at this station. It is the usual practice in earth-current measurements to record the changes in potential across two perpendicular lines $(1,000 \mathrm{ft}$ is a convenient length as the disturbances are of the order of millivolts per kilometer), so that any disturbance from the normal potential gradient may be treated vectorially. Within the limit of time resolution possible in these measurements, which were made with a paper speed of $4 \mathrm{in} . / \mathrm{hr}$, it was found that events could be readily correlated from all field points to Meanook. The average magnitude of a disturbance, as compared to that recorded at Meanook, varied from station to station, as shown on figure 2. These average mag-

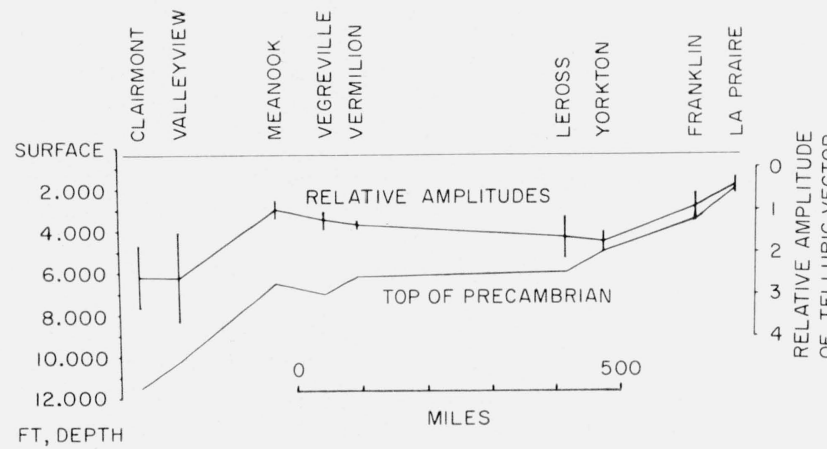

Figure 2. Ratio of telluric disturbance vector at Meanook to that at other stations, and depth of Precambrian basement. Vertical lines indicate standard deviations. nitudes were obtained by forming the resultant of the two component measurements for a number of disturbances, of period $1 \mathrm{~min}$ and greater, observed during several hours of recording at each point. On the same diagram is plotted the depth to the crystalline Precambrian basement, which is formed of rock of much higher resistivity than the overlying sedimentary rocks. There is a fairly good correlation between the two profiles, suggesting that the magnitude of disturbance of the electric field at any point is inversely proportional to basement depth. This would indeed be the case if source effects were completely uniform at all points, and if the basement were completely nonconducting. For then, if $h$ is the basement depth, and $\sigma$ the uniform conductivity of the sedimentary rocks

$$
\sigma h E=I
$$

where $I$ is the constant total current flowing in a strip of unit width. At the relatively low frequencies considered here the skin depth is much greater than any basement depth encountered. It was concluded from these results that the major reason for the electric field variation with location was change in effective conductivity, and that source distance effects were minor. The approach employed here is an application, on a very large scale, of a method of geophysical exploration first described by Migaux [4] as the telluric method. It should be noted from figure 1 that the stations were located very nearly
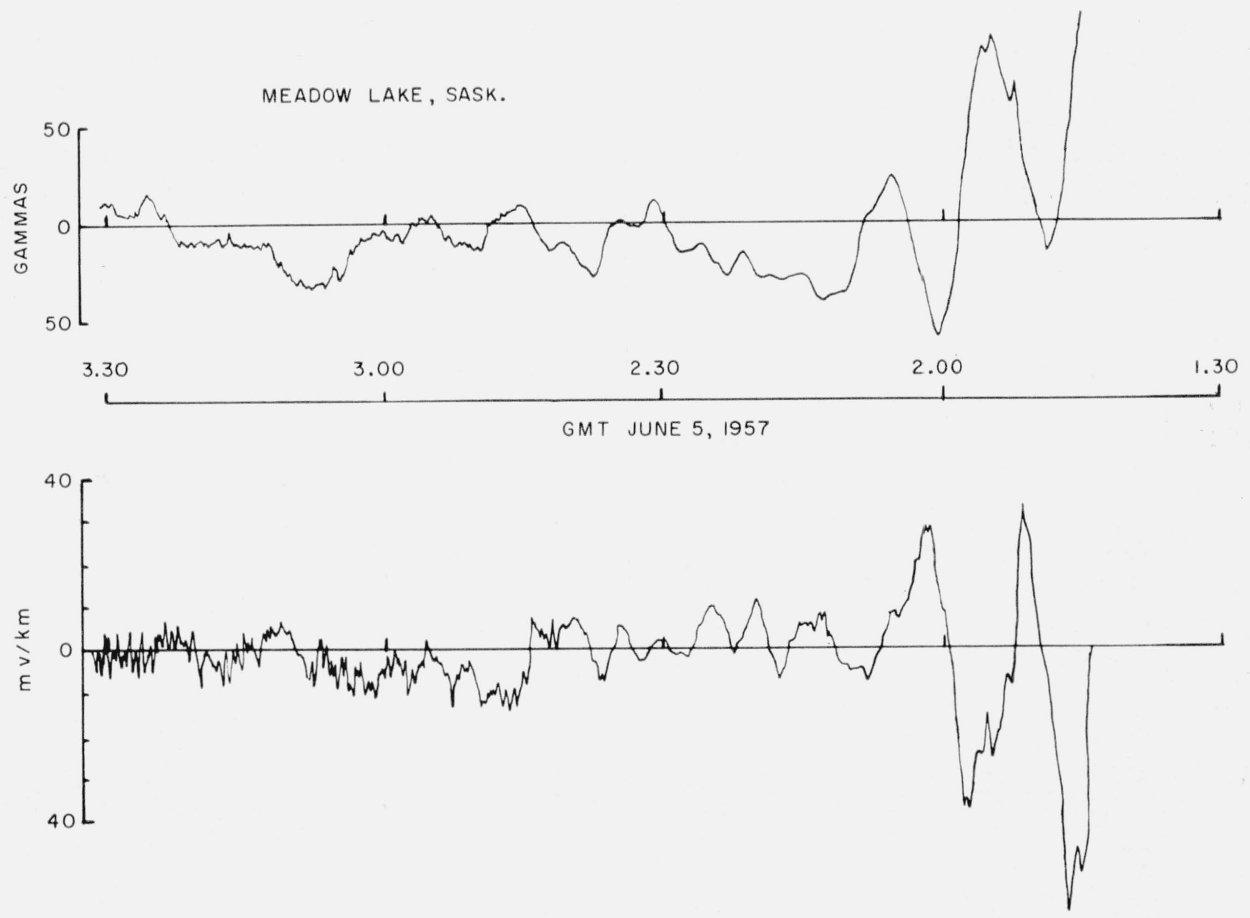

Figure 3. Portion of simultaneous magnetic and telluric records. 
along a line of constant magnetıc latitude, and different results could well be expected for points at different distances from the auroral zone.

\section{Magneto-Telluric Investigations}

The apparent uniformity of the natural electric field suggested that Cagniard's approach to determine conductivity at various depths could be useful. Simultaneous measurements, as in figure 3 , of electric and magnetic field variations were made at points where the depth to the resistant Precambrian rocks was known. At the time these measurements were made, a flux-gate magnetometer with a limit of sensitivity of 1 gamma $\left(10^{-5}\right.$ oersted) was used, and all recordings were made with pen recorders. The principle involved in Cagniard's analysis is the extraction of a value of apparent conductivity, by eq (1), for a series of disturbances of different periods. Because of the variation in depth of penetration with period, the apparent conductivity determined will be a function of period, and the form of this functional relationship will depend on the variation of conductivity with depth. Theoretical curves of apparent conductivity against period have been computed for cases of simple layering by Cagniard. It is apparent that the greatest labor involved is in the analysis of the records into discrete frequency components.

In future observations, it is intended that this analysis will be made by digital recording and machine computation. However, the values of apparent resistivity plotted logarithmically against period in figure 4 were obtained by Fourier analysis of the visual records. On the diagram are plotted also two theoretical curves for a two-layer structure. The upper layer is taken to be the sedimentary section, whose thickness is about $1.5 \mathrm{~km}$ at this location, with

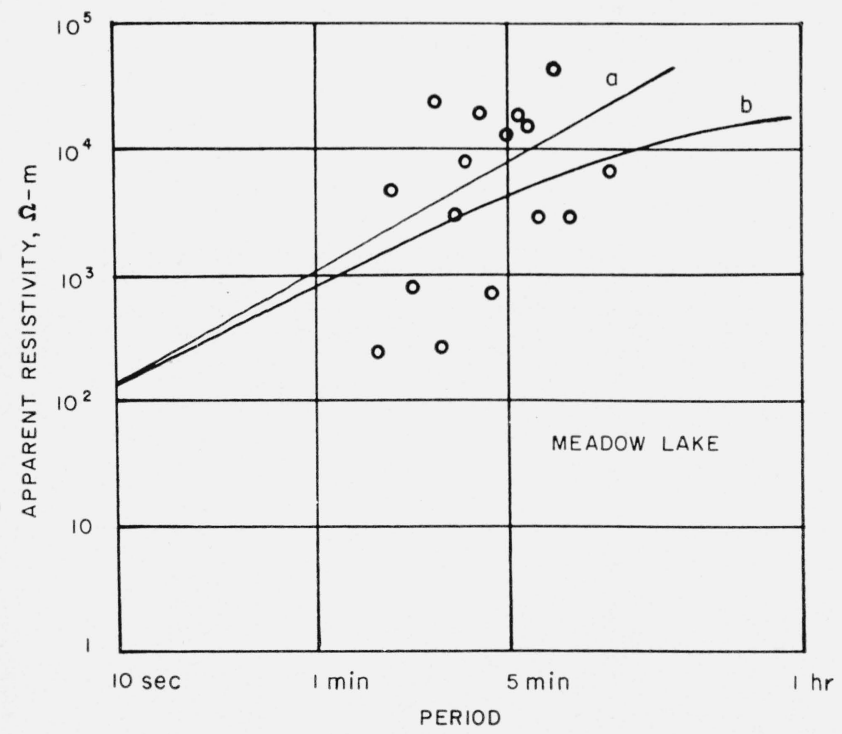

FIGURE 4. Apparent resistivity by magneto-telluric analysis as a function of period.

The curves (a) and (b) are the theoretical two-layer variations for basement resistivities of infinity and $30 \times 10^{4}$ ohmmeters, respectively. a resistivity, estimated from well $\operatorname{logs}$, of $30 \mathrm{ohm}-\mathrm{m}$. Since the scales are logarithmic, small errors in these assumptions affect the position, but not the form, of the curves. The two theoretical curves are plotted for basement resistivities of $30 \times 10^{4} \mathrm{ohm}-\mathrm{m}$ and infinity, respectively. While there is considerable scatter in the observed points, the general increase of apparent resistivity with period is indicated. In particular, there is a good indication that the basement resistivity is at least $30 \times 10^{5} \mathrm{ohm}-\mathrm{m}$. The observed points indicate a rate of increase of apparent resistivity greater than the theoretical curves, which suggests that the two-layer case is an oversimplification. However, it does appear that an analysis of magnetic and electric fields of this type, especially with more adequate separation of the frequency components, will provide useful information on crystal conductivities. Niblett [5] has extended the analysis to longer periods, using records from the Meanook observatory, and has detected an increase in conductivity at the base of the crust.

\section{Vertical Earth Currents}

There is some interest in observing the vertical component of electric field within the earth, because this will indicate the amount of deflection of the horizontal currents by local variations in conductivity, and also because of possible correlations with atmospheric measurements. Electrodes at depths of 800 and $3,800 \mathrm{ft}$ have been placed in the well shown in figure 1 , and a record of the potential gradient changes is available for a period of about $1 \mathrm{yr}$. Disturbances in the vertical component amounting to a few percent of the horizontal electric field have been measured, but the ratio of vertical to horizontal field shows a strong dependence on the azimuth of the horizontal current flow. At this particular location, the vertical component is greater when the disturbance current flows north-south, as indicated by the plot of figure 5. This strongly

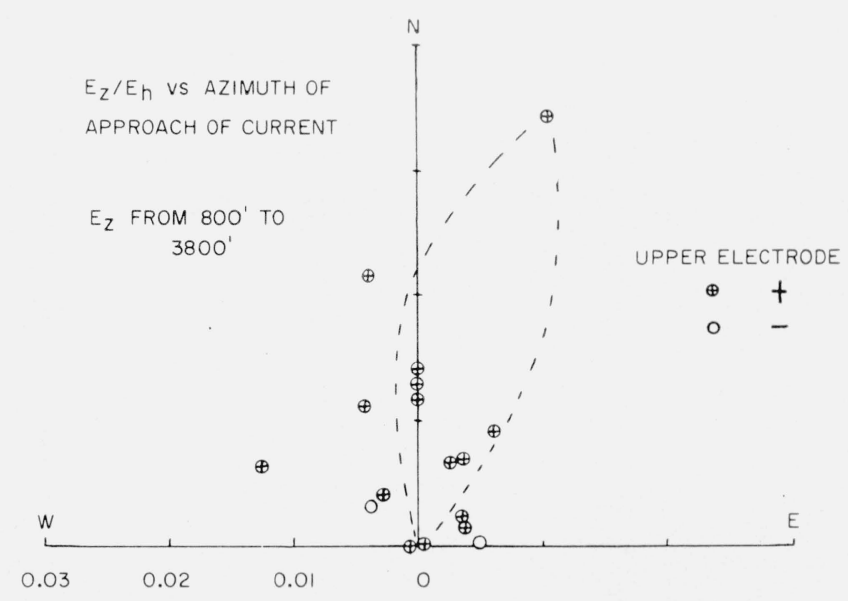

FiguRE 5. Ratio, obtained from well observations, of vertical to horizontal electric field, plotted radially, as a function of azimuth of horizontal flow, for a series of pulsations from the northerly half-circle. 
suggests lensing of the horizontal currents by a body of anomalous conductivity north or south of the observation well. However, a portion of the observed vertical electric field component in the earth may be associated with the earth-atmosphere current. As the normal component of current density is continuous across the surface, values of $6 \times 10^{13}$ ohm-m and $30 \mathrm{ohm}-\mathrm{m}$, respectively, for air and earth resistivities indicate a gradient in the earth only $5 \times 10^{-13}$ times the atmospheric gradient. During a period of atmospheric disturbance, however, the potential gradient in the air may be many times the fair-weather value of 100 $\mathrm{v} / \mathrm{m}$, and the corresponding effect in the earth may be detectable. It is hoped that simultaneous measurements in three components, in the earth, and measurements in the atmosphere at this site will indicate the manner in which the air-earth current flows into, or out of, the earth.

\section{References}

[1] V. P. Hessler and E. M. Wescott, Correlation between earth-current and geomagnetic disturbance. Nature 184, 627 (1959).

[2] L. Cagniard, Basic theory of the magneto-telluric method of geophysical prospecting, Geophysics 18, 605 (1953).

[3] J. R. Wait, On the relation between telluric currents and the earth's magnetic field, Geophysics 19, 281 (1954).

[4] L. Migaux, Une methode nouvelle de géophysique appliquée, Ann. géophys. 2, 131 (1946).

[5] E. R. Niblett, Variation of electrical conductivity with depth by the magneto-telluric method (abst.), Geophysics 24, 1150 (1959). 